\title{
SEMICLASSICAL THEORY FOR LOW DENSITY PROPERTIES OF POLAR HARD D-SPHERE FLUID MIXTURES
}

\author{
Shyambhu Kumar and Ranjit Prasad Yadav \\ Department of Physics, TU, Thakur Ram Multiple Campus, Birgunj, Nepal \\ E-mail: yadavranjeetprasad@gmail.com
}

\begin{abstract}
In the present paper, expansions are obtained for density-independent pair distribution function and second virial coefficient for D-dimensional molecular fluid mixtures of dipolar hard D-spheres in the semiclassical limit. Numerical results for the second virial coefficient are also estimated for binary mixture of (i) hard spheres and dipolar hard spheres and (ii) hard discs and dipolar hard discs. It is found that the quantum effects increase with increase of dipole moment $\mu_{2}$ as well as the concentration $x_{2}$. The purpose of the present work is to develop a theory for calculating the low density properties of the polar hard $D$-sphere fluid mixture in the semiclassical limit. We have also developed the theory for estimating the quantum corrections to the density independent pair distribution function (PDF) and second virial coefficient of the polar D-sphere fluid mixtures.
\end{abstract}

\section{Key words}

Semiclassical; quantum corrections; equilibrium properties; fluid mixtures

\section{Introduction}

We have developed a theory for calculating the quantum corrections to the low density equilibrium properties of polar hard $D$-sphere fluid mixtures with $D=2$ and 3 in the semiclassical limit. Equilibrium behaviour of pure polar hard sphere fluid $(D=3)$ and their mixtures is of great importance. A considerable progress has been made for classical fluid and fluid mixtures of hard sphere molecules with additional electrostatics. Gokhul \& Sinha (1997) have calculated the virial equation of state for classical polar hard D-sphere fluid mixtures with $\mathrm{D}=2$ and 3. Rai et al. (1990) have calculated the first quantum corrections to the thermodynamics of polar hard sphere fluids and fluid mixtures.

Moreover, we have also developed the theory for estimating the quantum corrections to the density independent pair distribution function (PDF) and second virial coefficient of the polar D-sphere fluid mixtures. 


\section{Potential model}

We have considered a D-dimensional fluid mixture of hard D-sphere with embedded central point dipoles. In this case the pair potential is given by

$\mathrm{u}_{\mathrm{ab}}\left(\mathrm{r} \omega_{1} \omega_{2}\right)=\mathrm{u}_{a b} h c_{(\mathrm{r})}+\mathrm{u}_{a b} d d\left(\mathrm{r} \omega_{1} \omega_{2}\right)$

Where $\mathrm{u} a c_{\mathrm{b}}$ is the hard sphere (or hard disc) potential

$$
\mathrm{u}_{a b} h c_{(\mathrm{r})}=\quad \infty \quad, \mathrm{r}<\sigma_{\mathrm{ab}}
$$

(2)

$$
=\quad \mathrm{o} \quad, \mathrm{r}>\sigma_{\mathrm{ab}}
$$

and $\mathrm{u} a d$ is the dipole-dipole interaction given by $\mathrm{u}_{a b}^{d d}\left(\mathrm{r} \omega_{1} \omega_{2}\right) \quad=\quad-\left(\mu_{\mathrm{a}} \mu_{\mathrm{b}} / \mathrm{r}^{\mathrm{D}}\right) \delta_{\mathrm{D}}(1,2)$

where $\mu_{\mathrm{a}}$ is the dipole moment of a molecule of species a and $\delta_{\mathrm{D}}(1,2)$ is the angle-dependent part of interaction. For two-dimensional system $(\mathrm{D}=2) \delta_{\mathrm{D}}(1,2)$ is given by

$\delta_{\mathrm{D}}(1,2)=\quad \operatorname{Cos}\left(\theta_{\mathrm{a}}+\theta_{\mathrm{b}}-\theta_{\mathrm{ab}}\right)$

Where $\theta_{\mathrm{a}}, \theta_{\mathrm{b}}$ and $\theta_{\mathrm{ab}}$ are the angles that determine the orientation of molecules with respect to the line joining the centres of molecules. On other hand, $\delta_{\mathrm{D}}(1,2)$ for a three-dimensional system $(\mathrm{D}=3)$ is given by (Hirsch felder et al, 1954)

$\delta_{\mathrm{D}}(1,2)=\quad 2 \operatorname{Cos} \theta_{\mathrm{a}} \operatorname{Cos} \theta_{\mathrm{b}}-\operatorname{Sin} \theta_{\mathrm{a}} \operatorname{Sin} \theta_{\mathrm{b}} \operatorname{Cos} \phi_{\mathrm{ab}}$

Here $\theta_{\mathrm{a}}, \theta_{\mathrm{b}}$ and $\phi_{\mathrm{ab}}=\phi_{\mathrm{a}}-\phi_{\mathrm{b}}$ determine the orientation of the molecules with respect to the line joining the centres of molecules. In Eq. (2) $\sigma_{\mathrm{ab}}$ is the diameter between the hard D-spheres of species a and b. For unlike interactions, $\sigma_{\mathrm{ab}}$ is given by

$$
\sigma_{\mathrm{ab}}=\left(\sigma_{11}+\sigma_{22}\right) / 2
$$

\section{Density independent pair distribution functions for D-dimensional molecular fluid mixture}

In analogy to that of pure fluid, it is shown that the path distribution function $\mathrm{n}^{\mathrm{ab}}{ }_{2}\left(\mathrm{x}_{1}, \mathrm{x}_{2}\right)$, giving the probability of finding a molecules of species a at $x_{1}=r_{1} \omega_{1}$ another molecule of species $b$ at $x_{2}=r_{2} \omega_{2}$, can be expanded in power of density $\mathrm{p}_{a}=\mathrm{N}_{\mathrm{a}} / \mathrm{V}$

$$
n_{2}^{a b}\left(\mathrm{x}_{1}, \mathrm{x}_{2}\right) \quad=\rho_{\mathrm{a}} \rho_{\mathrm{b}} w_{2}^{a b}\left(\mathrm{x}_{1}, \mathrm{x}_{2}\right)+\mathrm{O}\left(\rho_{\mathrm{a}} \rho_{\mathrm{b}} \rho_{\mathrm{c}}\right)
$$

Where $w_{2}^{a b}\left(\mathrm{x}_{1}, \mathrm{x}_{2}\right)$ is the two particle Slater sum. In the low density limit, the pair distribution function (PDF) defined as

$\mathrm{g}_{\mathrm{ab}}\left(\mathrm{x}_{1}, \mathrm{x}_{2}\right)=n_{2}^{a b}\left(\mathrm{x}_{1}, \mathrm{x}_{2}\right) / \rho_{\mathrm{a}} \rho_{\mathrm{b}}$

is equal to the Slater sum $\mathcal{W}_{2}^{a b}\left(\mathrm{x}_{1}, \mathrm{x}_{2}\right)$. Thus the density independent PDF for D-dimensional system is defined as

$$
\mathrm{g}_{\mathrm{ab}}\left(\mathrm{x}_{1} \mathrm{x}_{2}\right)=\mathcal{W}_{2}^{a b}\left(\mathrm{x}_{1}, \mathrm{x}_{2}\right)=2 ! \lambda_{a b}^{2 D}\left(\mathrm{~g}_{\mathrm{a}}, \mathrm{g}_{\mathrm{b}}\right)^{-1} \sum_{\mathrm{r}} \psi_{x}^{*}\left(\mathrm{x}_{1}, \mathrm{x}_{2}\right) \exp \left(-\beta \stackrel{\Lambda}{H}_{2}\right) \underset{x}{\psi}\left(\mathrm{x}_{1}, \mathrm{x}_{2}\right)
$$


Where, $\left.\left.\stackrel{\Lambda}{H}_{2}=\sum_{a=1}^{2} \hbar^{2} / 2 m_{a} \sum_{i=1}^{2} \nabla_{r i}^{2}-\sum_{a=1}^{2} \hbar^{2} / 2\right\}_{a}\right) \sum_{i=1}^{2} \nabla_{w i}^{2}+u_{a b}\left(x_{1}, x_{2}\right)$

and $\Psi_{\mathrm{x}}\left(\mathrm{x}_{1}, \mathrm{x}_{2}\right)$ is a set of orthogonal two particle wave functions. Using the centre of mass and relative coordinate $\mathrm{R}$ and $\mathrm{r}=\left(\mathrm{r}_{1}-\mathrm{r}_{2}\right)$, we can write eq. (7) as

$\left.\mathrm{g}_{\mathrm{ab}}\left(\mathrm{x}_{1} \mathrm{x}_{2}\right)=2^{\mathrm{D} / 2} \lambda_{a b}^{D}\left(\mathrm{~g}_{\mathrm{a}}, \mathrm{g}_{\mathrm{b}}\right)^{-1} \sum_{x} \psi_{x}^{*}\left(\mathrm{r} \omega_{1} \omega_{2}\right) \exp [-\beta \stackrel{\Lambda}{H} r e l)\right] \Psi_{x}\left(r \omega_{1} \omega_{2}\right)$

(9)

Where, $\hat{H}_{r e l}=-\left(\hbar^{2} / \mathrm{m}_{\mathrm{ab}}\right) \nabla_{r}^{2}-\left(\hbar^{2} / 2 l_{a b}\right) \sum_{i=1}^{2} \nabla_{\omega i}^{2}+u_{a b}\left(r \omega_{1} \omega_{2}\right)$

(10)

is relative Haliltonian of two particles of species a and $b, m_{a b}=2 m_{a} m_{b} /\left(m_{a}+m_{b}\right)$ and $l_{a b}=$ $2 l_{a} l_{b}\left(l_{a}+l_{b}\right)$, where $m_{a}$ and $l_{a}$ are, respectively, the mass and moment of inertia of a molecule of species a. In eq. (9), $\beta=(\mathrm{KT})^{-1}$ ( $\mathrm{T}$ being the absolute temperature and $\mathrm{K}$ be the Boltzmann factor). In the classical limit, eq. (9) reduces to classical PDF $g_{a b}^{c}\left(r \omega_{1} \omega_{2}\right)$, given by

$$
g_{a b}^{c}\left(r \omega_{1} \omega_{2}\right)=\exp \left[-\beta u_{a b}\left(r \omega_{1} \omega_{2}\right)\right]
$$

(11)

In the semiclassical limit, for hard-core plus attractive tail potential, eq. (9) can be solved to give (Singh et al., 1991)

$$
\begin{gathered}
\mathrm{g}_{\mathrm{ab}}\left(\mathrm{r} \omega_{1} \omega_{2}\right)=\exp \left[-\beta \mathrm{u}_{\mathrm{ab}}\left(\mathrm{r} \omega_{1} \omega_{2}\right)\right]\left[\mathbf{1}+U_{a b}^{m}\left(r \omega_{1} \omega_{2}\right) \quad \mathrm{r} \geq \sigma_{\mathrm{ab}}\right. \\
=\mathrm{o}
\end{gathered}
$$

Where, $U_{a b}^{m}=U_{a b}^{m t r}+U_{a b}^{m, r o t}$

$$
\text { (13) }
$$

with

$$
U_{a b}^{m, t r}\left(r \omega_{1} \omega_{2}\right)=U_{a b}^{m, h c}(r)+U_{a b}^{P, t r}\left(r \omega_{1} \omega_{2}\right)
$$

$$
U_{a b}^{m, r o t}\left(r \omega_{1} \omega_{2}\right)=U_{a b}^{P, r o t}\left(r \omega_{1} \omega_{2}\right)
$$

Here $U_{a b}^{m, h c}$ is the 'modified' Ursell function of the hard D-sphere mixture, $U_{a b}^{P, t r}$ and $U_{a b}^{P, r o t}$ are the 'modified' Ursell functions arising from the translational and rotational contributions of perturbation potential, respectively. They are given by

$U_{a b}^{P, t r}\left(r \omega_{1} \omega_{2}\right)=-\left(\hbar^{2} \beta^{2} / 6 m_{a b}\right)\left[\nabla_{r}^{2} U_{a b}^{p}-(1 / 2) \beta\left(\partial u_{a b}^{p} / \partial r\right)^{2}+3\left(\partial u_{a b}^{p} / \partial r\right) \delta\left(r-\sigma_{a b}\right)\right.$

and

(16a)

$U_{a b}^{p, r o t}\left(r \omega_{1} \omega_{2}\right)=-\left(\hbar^{2} \beta^{2} / 12 l_{a b}\right) \sum_{i=1}^{2}\left[\nabla_{\omega i}^{2} u_{a b}^{p}-(1 / 2) \beta\left(\nabla_{\omega i} u_{a b}^{p}\right)^{2}\right]$

(16b)

where, $(\partial / \partial r)\left[g_{a b}^{o h c}(r)=\delta\left(r-\sigma_{a b}\right)+0\left(\lambda_{a b} / \sigma_{a b}\right)\right.$

Here $\delta$ is the Dirac $\delta$ - function. In the semiclassical limit, $U_{a b}^{m, h c}$ is given by (Singh et al., 1991) $U_{a b}^{m, h c}(r)=-\exp \left[-\xi_{a b}^{2}\right]+[(D-1) / 2 \sqrt{2}]\left(\lambda_{a b} / \sigma_{a b}\right) \xi_{a b}^{2} \operatorname{erfec}\left(\xi_{a b}\right)$ 
Here $\xi_{a b}=\left[(2 \pi)^{1 / 2} /\left(\lambda_{a b} / \sigma_{a b}\right)\right]\left[\left(r / \sigma_{a b}\right)-1\right]$ and erfec $\left(\xi_{a b}\right)$ is the complementary error function.

The angle - averaged (density independent) PDF is defined as

$$
g_{a b}^{0}(r)=<g_{a b}^{0}\left(r \omega_{1} \omega_{2}\right)>\omega_{1} \omega_{2}
$$

Where, $<\ldots \ldots . .>\omega_{1} \omega_{2}=\Omega^{-2} \iint(\ldots) \omega_{1} \omega_{2}$

(19)

Substituting eq. (12) in eq. (18) and integrating over angles, we get

$$
\begin{aligned}
g_{a b}^{0}\left(r^{*}\right) & =<g_{a b}^{00}\left(r^{*}\right)+g_{a b}^{p, t r}\left(r^{*}\right)+g_{a b}^{p, r o t}\left(r^{*}\right)> & & \mathrm{r}^{*} \geq \sigma_{\mathrm{ab}} \\
& =\mathrm{o} & & \mathrm{r}^{*} \leq \sigma_{\mathrm{ab}}
\end{aligned}
$$

$(20)$

Where

$$
g_{a b}^{00}\left(r^{*}\right)=\left[1+u_{a b}^{m, h c}\left(r^{*}\right)\right]\left[1+\sum_{n=1}^{\infty}\left(a_{2 n}^{D} /(2 n) !\right)\left(\left(\mu_{a b}^{* 2}\right)^{2 n} / r^{* 2 D n}\right)\right]
$$

$$
g_{a b}^{p, t r}\left(r^{*}\right)=\left(\left(\lambda_{a b} / \sigma_{a b}\right)^{2} / 24 \pi\right)\left[\left(4 D+D^{2}\right) r^{*^{2}}-\left(6 D / r^{*}\right) \delta\left(r^{*}-1\right)\right] \sum_{n=1}^{\infty}\left(a_{2 n}^{D} /(2 n-1) !\right)\left(\left(\mu_{a b}^{* 2}\right)^{2 n} / r^{* 2 D n}\right)
$$

$$
g_{a b}^{p, r o t}\left(r^{*}\right)=\left(\left(\lambda_{a b} / \sigma_{a b}\right)^{2} / 24 \pi I_{a b}^{*}\right) \sum_{n=1}^{\infty}\left(a_{2 n}^{D} /(2 n-1) !\right)\left(\left(\mu_{a b}^{*^{2}}\right)^{2 n} / r^{* 2 D n}\right)
$$

Where, $\mathrm{r}^{*}=\mathrm{r} / \sigma_{\mathrm{ab}}, \mu_{a b}^{*^{2}}=\beta \mu_{\mathrm{a}} \mu_{\mathrm{b}} / \sigma_{a b}^{D}, \lambda_{\mathrm{ab}}=\mathrm{l}_{\mathrm{ab}} / \mathrm{m}_{\mathrm{ab}} \sigma_{a b}^{2}$ and $a_{2 n}^{D}=<\delta_{D}^{2 n}(1,2)>\omega_{1} \omega_{2}$

The values of $a_{2 n}^{D}$ for $\mathrm{D}=2$ and 3 are reported by (Singh et al., 1991) for the first few values of $\mathrm{n}$.

\section{Second virial coefficient of polar hard D-sphere fluid mixture}

We consider the second virial coefficient for D-dimensional fluid mixture of hard D-spheres with embedded central point dipoles. In this case pair potential is given by eq. (1).

The second virial coefficient for the $\mathrm{D}$-dimensional fluid mixture is given by

$\mathrm{B}(\mathrm{D})=-(1 / 2) \sum_{a, b} x_{a} x_{b} \int d r_{2}<\left[g_{a b}\left(r \omega_{1} \omega_{2}\right)-1\right]>\omega_{1} \omega_{2}$

(24)

Where $g_{a b}\left(r \omega_{1} \omega_{2}\right)$ is the density independent PDF of the semiclassical molcular fluid mixture of species $a$ and $b$, and $x_{a}=N_{a} / N$ is the concentration of species a. For a polar hard D-sphere fluid mixture, gab $\left(\mathrm{r} \omega_{1} \omega_{2}\right)$ is given by eq. (12)

Substituting eq. (12) in eq. (24), we obtain an expression for $B(D)$ in the form

$$
\mathrm{B}(\mathrm{D})=\mathrm{B}^{\mathrm{c}}(\mathrm{D})+\left[\mathrm{B}^{\mathrm{qc}}(\mathrm{D})\right]_{\mathrm{tr}}+\left(\mathrm{B}^{\mathrm{qc}}(\mathrm{D})\right]_{\mathrm{rot}}
$$

$$
\text { (25) }
$$

Where, 


$$
\begin{aligned}
& \mathrm{B}^{\mathrm{c}}(\mathrm{D})=-(1 / 2) \sum_{a, b} x_{a} x_{b} \int d r_{2}<\exp \left[-\beta u_{a b}\left(x_{1} x_{2}\right)\right]-1>\omega_{1} \omega_{2} \\
& (26) \\
& {\left[\mathrm{B}^{\mathrm{qc}}(\mathrm{D})\right]_{\mathrm{tr}}=-(1 / 2) \sum_{a, b} x_{a} x_{b} \int d r_{2}<\exp \left[-\beta u_{a b}\left(x_{1} x_{2}\right)\right] U_{a b}^{m, t r}\left(x_{1} x_{2}\right)>\omega_{1} \omega_{2}}
\end{aligned}
$$

$$
\left[\mathrm{B}^{\mathrm{qc}}(\mathrm{D})\right]_{\mathrm{rot}}=-(1 / 2) \sum_{a, b} x_{a} x_{b} \int d r_{2}<\exp \left[-\beta u_{a b}\left(x_{1} x_{2}\right) U_{a b}^{m, r o t}\left(x_{1} x_{2}\right)>\omega_{1} \omega_{2}\right.
$$

Here $B^{c}(D)$ is the classical second virial coefficient of the polar hard D-sphere fluid mixture, $\left[\mathrm{B}^{\mathrm{qc}}(\mathrm{D})\right]_{\mathrm{tr}}$ and $\left[\mathrm{B}^{\mathrm{qc}}(\mathrm{D})\right]_{\text {rot }}$ are the contribution to the quantum corrections to the second virial coefficient due to the translational and rotational parts, respectively. Here $<(\ldots)>\omega_{1} \omega_{2}$ is given by eq. (19), where

$$
\begin{aligned}
\mathrm{d} \omega_{\mathrm{i}} & =\mathrm{d} \theta_{\mathrm{i}} & & \text { for } \mathrm{D}=\mathbf{2} \\
& =\operatorname{Sin} \theta_{\mathrm{i}} \mathrm{d} \theta_{\mathrm{i}} \mathrm{d} \varphi & & \text { for } \mathrm{D}=3
\end{aligned}
$$

and for linear molecule, $\Omega=2 \pi$ for $D=2$ and $\Omega=4 \pi$ for $D=3$

Gokhul \& Sinha have calculated the classical second virial coefficient of dipolar hard D-sphere, fluid mixture (with $\mathrm{D}=\mathbf{2}$ and 3 ). Thus the result is

$\mathrm{B}^{\mathrm{c}}(\mathrm{D})=\left(\pi^{\mathrm{D} / 2} / 2 \Gamma(1+\mathrm{D} / 2)\right) \sum_{a, b} x_{a} x_{b} \sigma_{a b}^{D}\left[1-\sum_{n=1}^{\infty}\left(a_{2 n}^{D} /(2 n) !(2 n-1)\right)\left(\mu_{a b}^{*}\right)^{2 n}\right]$

Where $\Gamma(1)$ is the Gamma function. eq. (29) is an exact expression for $B^{c}(D)$ with $D=2$ and 3. This is monatonically decreasing function of $\mu_{a b}^{*}$.

In order to evaluate eq. (27), we make use of a Taylor expansion of $\mu_{a b}^{d d}\left(\mathrm{r} \omega_{1} \omega_{2}\right)$ about $\sigma_{\mathrm{ab}}$ and substitute eq. (14) in eq. (27), we get the following expression for $\left[\mathrm{B}^{\mathrm{qc}}(\mathrm{D})\right]_{\mathrm{tr}}$ for dipolar hard D-sphere fluid mixture.

$\left[\mathrm{B}^{\mathrm{qC}}(\mathrm{D})\right]_{\mathrm{tr}}=\left(\pi^{\mathrm{D} / 2} / 2 \Gamma(1+\mathrm{D} / 2)\right)$

$$
\left.\left.\sum_{a, b} x_{a} x_{b}^{+} \sigma_{a b}(D)(D / 2) / \sqrt{2}\right)\left(B_{a b}^{l l}(D)\right)_{t r}\left(\lambda_{a b} / \sigma_{a b}\right)^{2}+\ldots\right]
$$

Where,

$$
\begin{gathered}
\left(B_{a b}^{l}(D)\right)_{t r}=<\exp \left[-\beta \mu_{a b}^{d d}\left(\sigma_{a b} \omega_{1} \omega_{2}\right)>\omega_{1} \omega_{2}\right. \\
(31) \\
\left(B_{a b}^{l l}(D)\right)_{t r}=<\exp \left[-\beta \mu_{a b}^{d d}\left(\sigma_{a b} \omega_{1} \omega_{2}\right)>\omega_{1} \omega_{2}-\left(1 / 2(D-1)<\beta \sigma_{a b} \mu_{a b}^{d d^{\prime}}\left(\sigma_{a b} \omega_{1} \omega_{2}\right)\right]\right. \\
(32) \\
\exp \\
\left.\beta \mu_{a b}^{d d}\left(\sigma_{a b} \omega_{1} \omega_{2}\right)\right]>\omega_{1} \omega_{2}+(1 / 4(D-1)) \beta^{2} \int_{1}^{\infty} d r * r^{*-1}<\left(\partial u_{a b}^{d d}\left(r^{*} \omega_{1} \omega_{2}\right) / \partial r^{*}\right)^{2} \\
\quad \exp \left[-\beta u_{a b}^{d d}\left(r^{*} \omega_{1} \omega_{2}\right)\right]>\omega_{1} \omega_{2}
\end{gathered}
$$


Where, $u_{a b}^{d d^{\prime}}=\partial u_{a b}^{d d} / \partial r$ and $\mathrm{r}^{*}=\mathrm{r} / \sigma_{\mathrm{ab}}$

Similarly substituting eq. (15) in eq. (28), we get

$$
\left[\mathrm{B}^{\mathrm{qc}}(\mathrm{D})\right]_{\mathrm{rot}}=\left(\pi^{\mathrm{D} / 2} / 2 \Gamma(1+\mathrm{D} / 2)\right) \sum_{a, b} x_{a} x_{b} \sigma_{a b}^{D}\left[\left(D(D-1) / 6 \pi l_{a b}^{*}\right)\left(B_{a b}^{l l}(D)\right)_{r o t}\left(\lambda_{a b} / \sigma_{a b}\right)^{2}+\ldots\right]
$$

Where,

$$
\left.B_{a b}^{l l}(D)\right)_{r o t}=(1 / 4(D-1)) \beta \int_{1}^{\infty} d r^{*} r^{* D-1}<\nabla_{\omega i}^{2} u_{a b}^{d d}\left(r^{*} \omega_{1} \omega_{2}\right) \exp \left[-\beta u_{a b}^{d d}\left(r * \omega_{1} \omega_{2}\right)\right]>\omega_{1} \omega_{2}
$$

and $l_{a b}^{*}=l_{a b} / m_{a b} \sigma_{a b}^{2}$

Finally eq. (25) can be written as

$$
\begin{aligned}
\mathrm{B}(\mathrm{D})= & \mathrm{B}^{\mathrm{c}}(\mathrm{D})+\left(\pi^{\mathrm{D} / 2} / 2 \Gamma(1+\mathrm{D} / 2)\right) \sum_{a, b} x_{a} x_{b} \sigma_{a b}^{D}\left[( d / 2 \sqrt { 2 } ) \left(B_{a b}^{l}(D)\left(\lambda_{a b} / \sigma_{a b}\right)\right.\right. \\
& \left.+(\mathrm{D}(\mathrm{D}-1) / 6 \pi)\left(B_{a b}^{l l}(D)\right)\left(\lambda_{a b} / \sigma_{a b}\right)^{2}+\ldots\right]
\end{aligned}
$$

Where, $B_{a b}^{l}(D)=\left(B_{a b}^{l}(D)\right)_{t r}$

$$
B_{a b}^{l l}(D)=\left(B_{a b}^{l l}(D)\right)_{t r}+\left(l_{a b}^{*}\right)^{-1}\left(B_{a b}^{l l}(D)\right)_{r o t}
$$

(37)

Thus like one component fluid the first order quantum correction arises from the translational part only where as the second order quantum correction is due to the translational and rotational contributions.

In case $u_{a b}^{d d}=0$, we have

$$
\begin{aligned}
& B_{a b}^{l}(D)=1 \\
& B_{a b}^{l l}(D)=\left(B_{a b}^{l l}(D)\right)_{t r}=1
\end{aligned}
$$

Which are correct for hard D-sphere fluid mixture (Mishra \& Sinha, 1985)

For the dipole-dipole potential model given by eq. (3), the quantum correction terms are given by

$$
\begin{gathered}
\left(B_{a b}^{l}(D)\right)_{r t}=1+\left(a_{2}^{D} / 2\right)\left(\mu_{a b}^{* 2}\right)^{2}-\left(a_{4}^{D} / 24\right)\left(\mu_{a b}^{* 2}\right)^{4}+\left(a_{6}^{D} / 720\right)\left(\mu_{a b}^{* 2}\right)^{6} \\
\left(B_{a b}^{l l}(D)\right)_{t r}=1+\left(\left(D^{2}-2 D-4\right) / 4(D-1)(D+2)\right) a_{2}^{D}\left(\mu_{a b}^{*^{2}}\right)^{2}-\left((10 D-4) / 48(D-1)(3 D+2) a_{2}^{D}\left(\mu_{a b}^{*^{2}}\right)^{4}\right. \\
\quad+\left(\left(5 D^{2}+18 \mathrm{D}+4\right) / 1440(\mathrm{D}-1)(5 \mathrm{D}+2)\right) a_{6}^{D}\left(\mu_{a b}^{*^{2}}\right)^{4}+\ldots
\end{gathered}
$$

and 


$$
\left(B_{a b}^{l l}(D)\right)_{r o t}=\left(a_{2}^{D} / 4 D\right)\left(\mu_{a b}^{*^{2}}\right)^{2}-\left(a_{4}^{D} / 72 D\right)\left(\mu_{a b}^{*^{2}}\right)^{4}+\left(a_{6}^{D} / 2400 D\right)\left(\mu_{a b}^{*^{2}}\right)^{6}+\ldots
$$

We calculate these coefficients using Pade' approximant constructed with last two terms in each series.

We consider a binary mixture of hard D-sphere (Species 1) and dipolar hard D-sphere (species 2) having the same diameters i.e. $\sigma_{11}=\sigma_{22}=\sigma$. Further we assumed that molecules of species 1 and 2 interact via hard D-sphere potential. In this case the hard D-sphere is taken as the reference system. Then eq. (36) reduces to

$$
\mathrm{B}(\mathrm{D})=\mathrm{B}^{\mathrm{c}}(\mathrm{D})+B_{H C}^{c}(D)\left[(D / 2 \sqrt{2})\left(B^{1}(D)\right)(\lambda / \sigma)+\left(D(D-1 / 6 \pi) B^{11} D\right)(\lambda / \sigma)^{2}+\ldots\right]
$$

(41)

Where

$$
\begin{aligned}
\mathrm{B}^{1}(\mathrm{D})= & 1+x_{2}^{2}\left[B_{22}^{l}(D)-1\right] \\
& (42 \mathrm{a}) \\
\mathrm{B}^{\mathrm{I1}}(\mathrm{D})= & 1+x_{2}^{2}\left[B_{22}^{l l}(D)-1\right] \\
& (42 \mathrm{~b})
\end{aligned}
$$

(Baus \& Colot, 1987)

$$
B_{H C}^{c}(D)=\left[\pi^{D / 2} / 2 \Gamma(1+D / 2)\right] \sigma^{D}
$$

is the classical second virial coefficient of the hard D-sphere fluid.

Table 1 shows the magnitudes of the quantum correction coefficients for a binary mixtures of hard D- spheres and dipolar hard D-spheres (with D = 2 and 3) having the same diameter as a function of $\mu^{* 2} \equiv \mu_{22}^{* 2}$ for $\mathrm{x}_{2}=0.5$ and 1.0. The values of $\mathrm{B}^{1}(\mathrm{D})$ and $\mathrm{B}^{11}(\mathrm{D})$ decrease with decrease of $\mathrm{x}_{2}$ and reduces to unity $\mathrm{x}_{2}=$ o.o. Further we see that the second order quantum correction depends on the values of $\mu^{* 2}$ and $l^{*}$, which the second order quantum correction depends only on the value of $\mu^{* 2}$.

The second virial coefficient $B^{*}(D)=\left(B(D) / \sigma^{D}\right)$ for the binary mixture of hard discs and dipolar hard spheres (HS-DHS) are reported in Figs. 1 and 2, respectively, as a function of

\begin{tabular}{|c|c|c|c|c|c|c|c|}
\hline \multirow{2}{*}{$D$} & \multirow{2}{*}{$\mu^{*^{2}}$} & \multicolumn{3}{|c|}{$x_{2}=0.5$} & \multicolumn{3}{|c|}{$x_{2}=1.0$} \\
\hline & & $B_{t r}^{1}$ & $\mathbf{B}_{t r}^{I I}$ & $B_{r \alpha}^{\prime \prime}$ & $B_{t r}^{\prime}$ & $\mathrm{B}_{\mathrm{tr}}^{11}$ & $B_{r a t}^{\prime 1}$ \\
\hline \multirow[t]{4}{*}{2} & 0.0 & 1.000 & 1.000 & 0.0 & 1.000 & 1.000 & 0.0 \\
\hline & 1.0 & 1.067 & 0.821 & 0.033 & 1.266 & 0.285 & 0.130 \\
\hline & 2.0 & 1.320 & -0.040 & 0.147 & 2.281 & -3.160 & 0.589 \\
\hline & 3.0 & 1.995 & -3.242 & 0.485 & 4.938 & -15.969 & 1.621 \\
\hline \multirow[t]{4}{*}{3} & 0.0 & 1.000 & 1:000 & 0.0 & 1.000 & 1.000 & 0.0 \\
\hline & 1.0 & 1.094 & 0.829 & 0.015 & 1.376 & 0.315 & 0.060 \\
\hline & 2.0 & 1.551 & -0.556 & 0.079 & 3.202 & -5.225 & 0.315 \\
\hline & 3.0 & 3.735 & -55.289 & 0.318 & 11.938 & -220.155 & 1.270 \\
\hline
\end{tabular}
$\mu^{* 2}$ with $1^{*}=0.07$ for $\mathrm{x}_{2}=0.5$ and 1.0 at $\lambda / \sigma=0.0$ and 0.1 . The case $\lambda / \sigma=0.0$ corresponds to the classical values. We find that the quantum effects which increase with increase of $\mu^{* 2}$, decrease with increase of $\mathrm{x}_{2}$.

Table 1: Quantum correction coefficients $B^{1,11}(D)$ for binary mixtures of hard D-spheres and dipolar hard $D$-spheres ( $D=2$ and 3 ) as a function of $\mu^{*^{2}}$ 


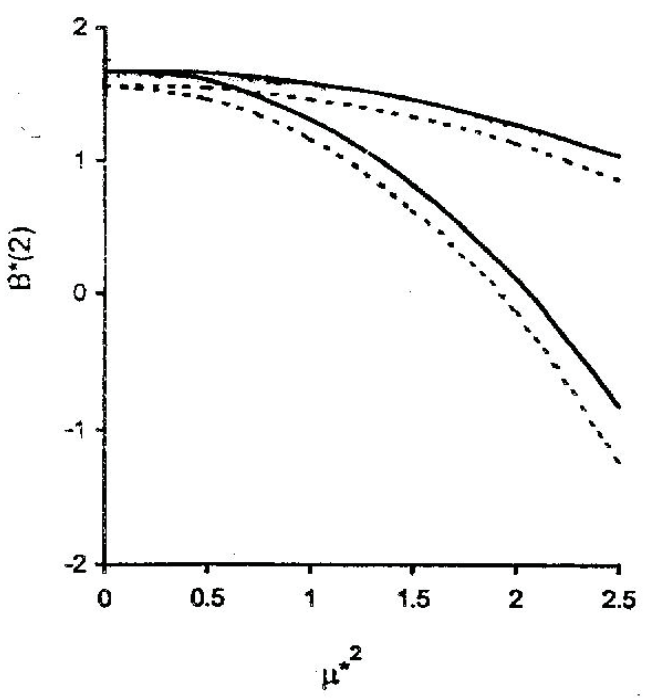

Fig.1 The second virial coefficient $\mathrm{B}^{*}(2)$ of HD-DHD mixture as a function of $\mu^{*^{2}}$ with $\mathrm{I}^{*}=$ 0.07 for $x_{2}=0.5$ and 1.0 at $\lambda / \sigma=0.0$ and 0.1 . The thick line represents $\lambda / \sigma=0.1$ and dotted line $\lambda / \sigma=0.0$

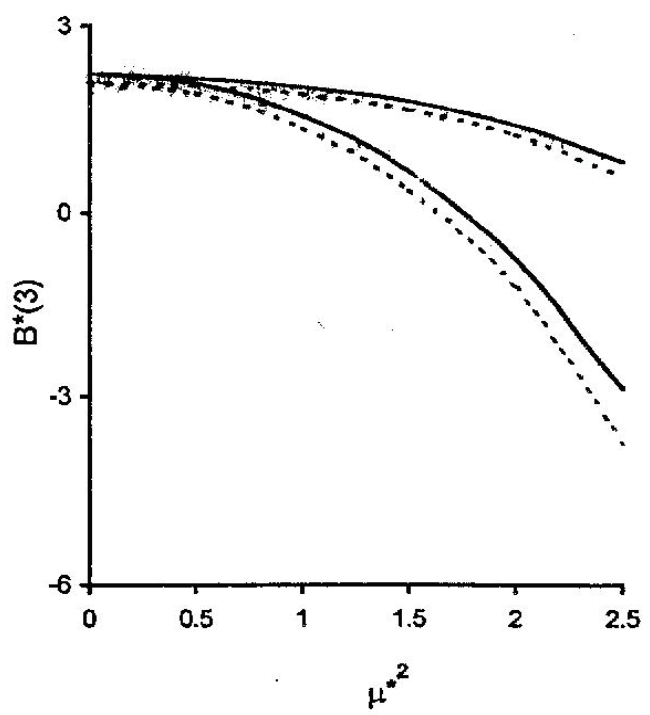

Fig.1 The second virial coefficient $\mathrm{B}^{*}(3)$ of HS-DHS mixture as a function of $\mu^{*}$ with $l^{*}=$ 0.07 for $x_{2}=0.5$ and 1.0 at $\lambda \sigma=0.0$ and 0.1 . Keys The Keys are same as Fig. 1.

\section{Conclusion}

From explicit expressions for the density independent PDF $\mathrm{g}_{\mathrm{ab}}\left(\mathrm{r} \omega_{1} \omega_{2}\right)$ and second virial coefficient for the dipolar hard D-sphere fluid mixture, we find that the quantum effects increase with increase of $\mu^{* 2}$ as well as $\mathrm{x}_{2}$.

\section{Acknowledgement}

We would like to acknowledge the contributions of other members of Department of Physics Thakur Ram Moltiple Campus, Birgunj without which this article would not have been possible.

\section{References}

Baus, M. \& Colot, J. L. (1987). Thermodynamics and structure of fluid of hard rods, disks, spheres or hyperspheres from rescaled virial expansions. Phys. Rev. A, 36, 2912-3925.

Gokhul, S. K. \& Sinha, S. K. (1997). Virial equation of state of polar hard D-sphere fluid mixture, Indian J. of Pure E App. Phys., 35, 499-507.

Hirsch felder, J.O., Curtiss, C.F. and Bird, (1954). Molecular theory of gases and liquids. New York : Wiley.

Mishra, B.M. and Sinha, S. K. (1985). Semiclassical statistical mechanics of two dimensional fluid mixture of hard discs, J. of Math. Phys., 26, 495-504.

Rai, B., Prasad, N. and Sinha, S. K. (1990). Quantum corrections to thermodynamics of polar hard sphere fluids. Pramana J. of Phys., 35, 533-555.

Singh, S. K., Prasad, N. and Sinha, S. K. (1991). Semiclassical statistical mechanics of simple molecular fluid one, equilibrium properties of dilute two and three dimensional fluid. Physica A, 179, 378-410. 